\title{
THE ANTIFUNGAL ACTIVITY OF ANTIBIOTIC XG ${ }^{1}$
}

\author{
By GLADYS L. HOBBY, PETER P. REGNA, NANCY DOUGHERTY, \\ AND WILLIAM E. STIEG
}

\section{(From the Biological and Chemical Research and Development Departments of Chas. Pfizer \& Co., Brooklyn, N.Y.)}

Lewis, Hopper, and Shultz, in 1946 (1), reported the existence of an antimicrobial agent in culture filtrates of an organism closely related to Bacillus subtilis, and demonstrated that this substance possesses a remarkable antifungal activity against Trichophyton gypseum and certain other pathogenic fungi, as well as against certain of the Gram-positive bacteria. During the following year, Lewis and Hopper undertook to investigate methods of producing this antibiotic on a small scale, and succeeded in recovering a sufficient quantity to permit preliminary study of its biological and chemical properties as well as its therapeutic efficacy in fungous infections in man. These early studies of Lewis and his associates suggested that Antibiotic XG may be a highly effective chemotherapeutic agent in the treatment of fungous infections in man.

The present report will discuss certain of the chemical and biological properties of this new antifungal agent. ${ }^{2}$

\section{NATURE OF ANTIBIOTIC}

Antibiotic XG is produced by the growth of an unidentified organism closely related to $B$. subtilis. The antibiotic may be absorbed from fermentation liquor onto carbon at neutral $\mathrm{pH}$ and may be eluted with aqueous acetone. It is soluble in methanol and ethanol. It is insoluble in ether, chloroform, ethyl acetate, and dry acetone.

Antibiotic XG is stable in broth when heated

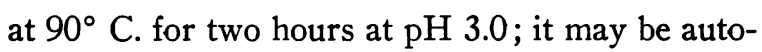
claved at 15 pounds pressure, for at least 10 minutes, at $\mathrm{pH} 7.0$ without significant loss in potency. In wet acetone, it is stable at room temperature

\footnotetext{
1 Presented at the Second National Symposium on Recent Advances in Antibiotics Research held in Washington, D. C., April 11-12, 1949, under the auspices of the Antibiotics Study Section, National Institutes of Health, Public Health Service, Federal Security Agency.

2 The preparations of Antibiotic XG used in this study were prepared in the Biochemical and Chemical Research and Development Departments of Chas. Pfizer \& Co., Inc., Brooklyn, N. Y.
}

for at least eight months, and in anhydrous alcohol, at a concentration of 3,500 units per cc., it is stable for at least two months.

Antibiotic $\mathrm{XG}$ is an amphoteric polypeptide which gels on standing in water. The gel form is biologically inactive but can be reactivated by dissolving in anhydrous solvents. The polypeptide may be hydrolyzed and on paper chromatography shows the presence of several different amino acids.

\section{METHODS AND RESULTS}

\section{Standardization}

The activity of Antibiotic XG is measured in terms of $T$. gypseum dilution units, one unit being the least amount of antibiotic which will inhibit the growth of a standard strain of $T$. gypseum under controlled conditions. The master standard used for all biological assays of Antibiotic XG has an activity of $70 \mathrm{~T}$. gypseum dilution units per $\mathrm{mg}^{3}$ Preparations having potencies as high as 250 units per $\mathrm{mg}$. have been obtained.

\section{In vitro activity}

Methods. The sensitivities of a wide variety of microorganisms to Antibiotic XG were determined in the following manner. Except when otherwise specified, a beef infusion broth (2) buffered at $\mathrm{pH} 7.8$ was used throughout. It was recognized that other mediums, such as Sabouraud's agar, are more commonly used for growth of the pathogenic fungi. It was felt advisable to use the beef infusion broth, however, in view of the fact that it appeared suitable for the growth of both the pathogenic bacteria and fungi.

In the case of all bacteria tested and in the case of Monilia albicans, 15-16 hour broth cultures were used except when otherwise indicated. In the case of other fungi than $M$. albicans, suspensions of six to eight day cultures on Sabouraud's agar were used. In all instances, bacterial cultures were diluted in broth to a constant density immediately prior to use. A density allowing $82-86 \%$ transmission on a Photovolt Lumetron No. 400 was arbitrarily chosen as standard. Suspensions of the fungous cultures were diluted to approximately the same

\footnotetext{
3 We are indebted to Dr. B. A. Sobin for all assay data reported herein.
} 
TABLE I

The Antimicrobial Action of Antibiotic XG Medium: Nutrient Broth

\begin{tabular}{l|c|c|c}
\hline \hline \multicolumn{1}{c|}{ Organisms } & $\begin{array}{c}\text { No serum } \\
\text { Sensitivity: } \\
\text { units/cc. }\end{array}$ & $\begin{array}{c}1 \% \% \\
\text { Rabbit } \\
\text { serum }\end{array}$ & $\begin{array}{c}5 \% \\
\text { Rabbit } \\
\text { serum }\end{array}$ \\
\hline Sporotrichum schenkii & 11.2 & 11.2 & 5.6 \\
Microsporum canis & 0.7 & 0.7 & 1.4 \\
Trichophyton rubrum & 11.2 & 2.8 & 1.4 \\
Cryptococcus necformans & 1.4 & 2.8 & 5.6 \\
Trichophyton gypseum & 2.8 & 1.4 & 0.7 \\
Epidermophyton floccosum & 2.8 & 1.4 & 0.7 \\
Microsporum audouini & 11.2 & 1.4 & 1.4 \\
Rhodotorula & 1.4 & 1.4 & 5.6 \\
Monilia albicans & $1.0 *$ & 90.0 & 90.0 \\
\hline
\end{tabular}

* On prolonged incubation sensitivity of this one organism frequently decreased to $90-100$ units per cc.

Sensitivity tests were carried out in broth and were read after two days' incubation. Sensitivity was accepted as the least amount of Antibiotic XG causing complete inhibition of growth.

density but clumping of the organisms prevented exact standardization by means of the Photovolt Lumetron.

A graded serial dilution technic was used in all tests. To each $1.8 \mathrm{cc}$. of serially diluted antibiotic, $0.2 \mathrm{cc}$. of a $10^{-4}$ dilution of bacterial culture or $0.2 \mathrm{cc}$. of an undiluted suspension of fungous culture was added. Incubation was carried out at $37^{\circ} \mathrm{C}$. The amount of growth was recorded at 24 and 48 hours in the case of bacteria and at two and five days in the case of fungi. The sensitivity of an organism was accepted as the least amount of Antibiotic XG which would cause complete inhibition of growth, as indicated by absence of gross turbidity. It was recognized, however, that this did not necessarily indicate a bactericidal level.

Results. Antibiotic XG is a highly efficient antifungal agent, active against all strains of pathogenic fungi tested. As shown in Table I, it is active in the presence of serum as well as in its

TABLE II

The antibacterial action of Antibiotic $X G$

\begin{tabular}{l|r|c}
\hline \multirow{2}{*}{ Organism } & \multicolumn{2}{|c}{ Sensitivity: units/cc, } \\
\cline { 2 - 3 } & 24 hours & 48 hours \\
\hline Streptococcus hemo. & 4.0 & 64 \\
Staphylococcus aureus & $>315$ & $>315$ \\
Diplococcus pneumoniae & 2.0 & 31.5 \\
Escherichia coli (BW41) & $>315$ & $>315$ \\
E. coli (W87) & $>315$ & $>315$ \\
Eberthella typhosa & $>315$ & $>315$ \\
Salmonella dys. & $>315$ & $>315$ \\
S. paratyphosa & $>315$ & $>315$ \\
Aerobacter aerogenes & $>315$ & $>315$ \\
Proteus vulgaris & $>315$ & $>315$ \\
Klebsiellia pneumoniae & $>315$ & $>315$ \\
Pseudomonas pyocyaneus & $>315$ & $>315$ \\
Brucella bronchiseptica & $<1.7$ & $>315$ \\
\end{tabular}

absence. In general, sensitivities varied little with time of incubation. In the case of $M$. albicans, growth was rapid in the medium used and in this instance sensitivity varied markedly depending upon the length of the incubation period.

The sensitivity of 13 strains of microorganisms, belonging to 10 different bacterial species, is shown in Table II. A temporary bacteriostatic effect was demonstrable against Streptococcus hemolyticus (Strain C203 Mv) and against Diplococcus pneumoniae (Strain I/230). All other bacteria tested were completely resistant to the action of Antibiotic XG, as prepared in our laboratories.

\section{Mechanism of action}

The early studies on the sensitivity of various microorganisms to Antibiotic XG indicated that this antimicrobial agent is strongly fungistatic in nature, and that its action may be affected markedly by changes in the number of organisms present, the concentration of the agent, the temperature of incubation, as well as other factors. To study the effect of Antibiotic XG on certain of the sporulating or slowly-growing pathogenic fungi, by means of growth curves such as are often used in the study of the action of antimicrobial agents on bacteria, seemed impractical. $M$. albi cans and a Rhodotorula, however, have lent themselves readily to this type of microbiological test.

Methods. Preparations of Antibiotic XG, ranging in potency from 65-100 units per $\mathrm{mg}$., were used throughout these experiments. Growth curves were carried out in the usual manner, as described in a previous communication (3). In certain experiments, all factors were held constant with the exception of the number of organisms; in other experiments, the concentration of drug or the temperature of incubation was varied. In all instances, the number of organisms per cc. at any given time during the incubation period was determined by the pour plate technic.

Effect of concentration of Antibiotic $X G$ on growth of certain pathogenic fungi. The effect of varying concentrations of Antibiotic XG on the growth of $M$. albicans, at $37^{\circ} \mathrm{C}$. and at $26^{\circ} \mathrm{C}$., is shown in Figures 1 and 2. At $37^{\circ} \mathrm{C}$., there was no growth of $M$. albicans when the initial number of organisms was in excess of $1,000,000$ organisms per cc. Concentrations of 500-1,000 units of Antibiotic XG were sufficient, however, to produce an apparent fungicidal effect when the initial number 


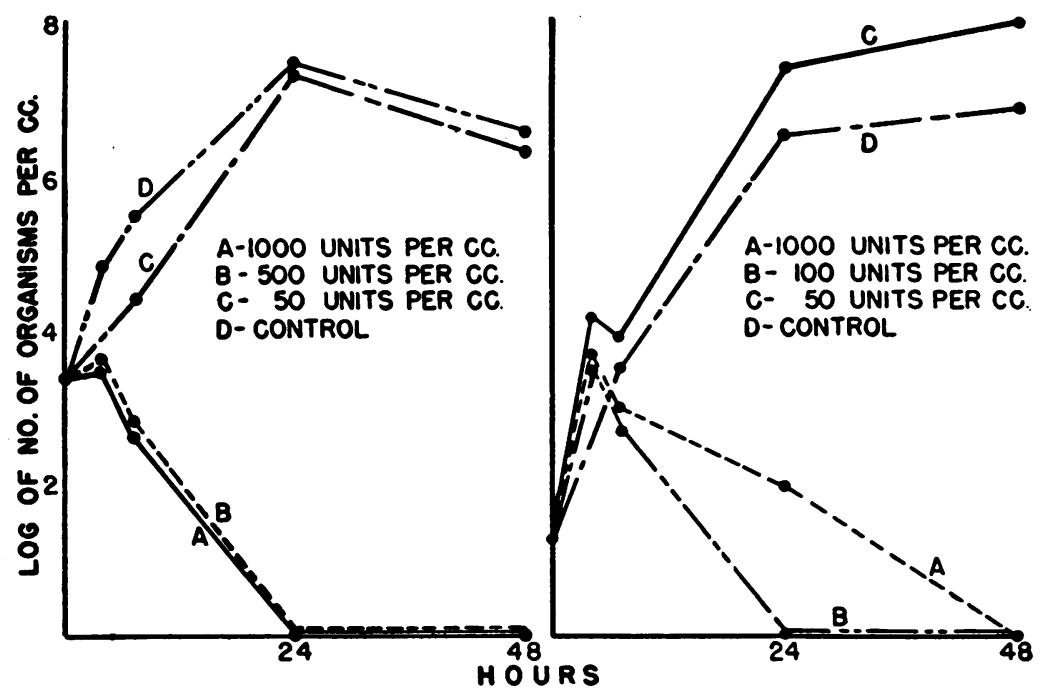

Fig. 1. The Effect of Varying Concentrations of Antibiotic XG on Growth of Monilia albicans $\left(37^{\circ} \mathrm{C}\right.$.)

of organisms was in the vicinity of 10,000 organisms per cc. With this number of organisms, 50 units per cc. produced no effect on the growth of the organism. With smaller numbers of organisms, $100-1,000$ units produced routinely an in- itial stimulation of growth followed by a gradual decrease in the number of organisms present. As with larger numbers of organisms, 50 units per cc. was totally ineffective.

The data presented indicate that growth of $M$.

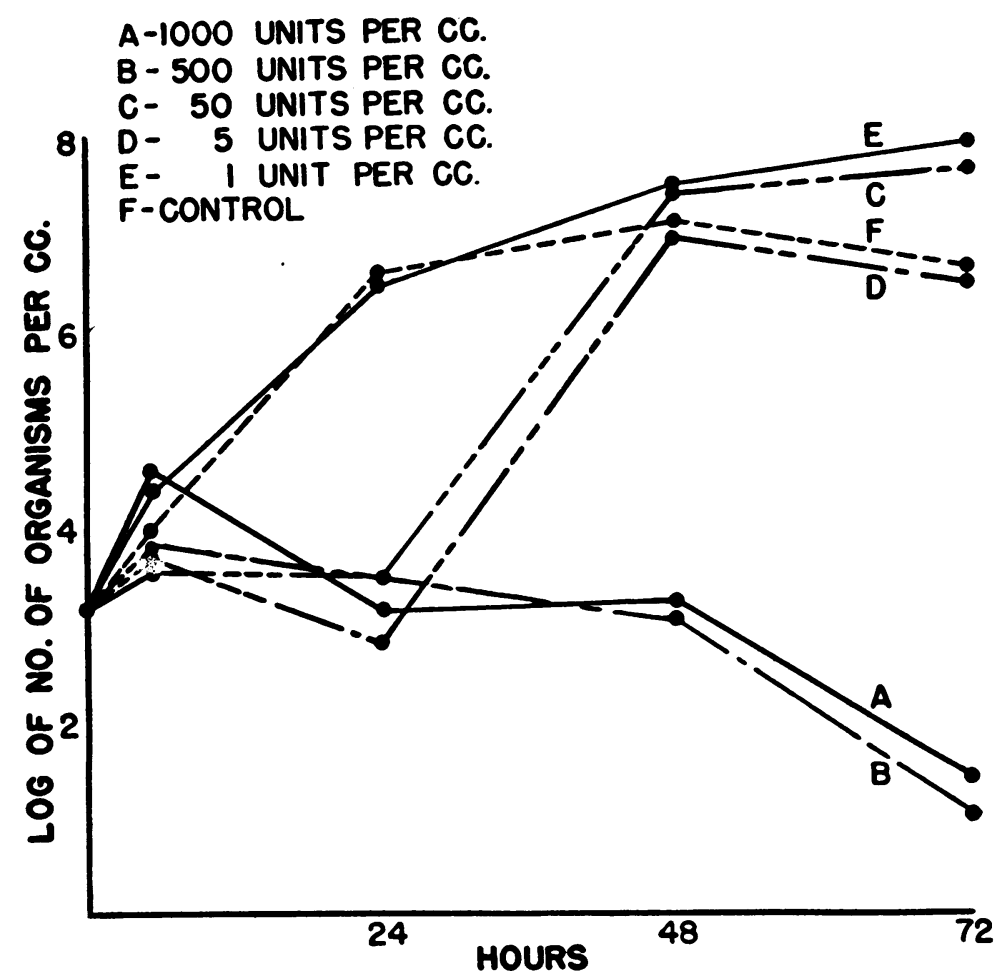

Fig. 2. The Effect of Varying Concentrations on Antibiotic XG on GrowrH or Monilia albicans $\left(26^{\circ} \mathrm{C}\right.$.) 
albicans in the medium used is more rapid at $37^{\circ}$ C. than at $26^{\circ} \mathrm{C}$. In like manner, the action of Antibiotic $\mathrm{XG}$ is more complete at $37^{\circ} \mathrm{C}$. than at $26^{\circ} \mathrm{C}$. No decrease in the number of organisms present was apparent in any instance unless there was also evidence of multiplication.

The effect of varying concentrations of Antibiotic XG on a more sensitive organism than $M$. albicans, namely a Rhodotorula, is shown in Figure 3. In this instance, the fungistatic action of Antibiotic XG was apparent even in the presence of an initial concentration of $1,000,000$ organisms per cc. Ten to 100 units per cc. was sufficient to produce a gradual decrease in the microbial count. Two units per cc. caused an inhibition of multiplication only, with no actual decrease in number of organisms, while 0.5 unit per cc. allowed full growth. It should be noted that in contrast to $M$. albicans, the Rhodotorula produced some multiplication in the absence of Antibiotic $\mathrm{XG}$, under the conditions of this experiment.

Effect of varying concentrations of organisms on the action of Antibiotic XG. In the presence of 1,000 units of antibiotic per cc. of medium, Antibiotic XG exerted a fungicidal action on $M$. albicans, provided incubation was carried out at $37^{\circ}$
C., and provided the initial number of organisms was less than 10,000 per cc. If incubation was carried out at $26^{\circ}$ C., a fungistatic effect was apparent, again providing the initial number of organisms was less than 10,000 per cc. With larger numbers of organisms, no fungistatic or fungicidal action on the part of the antibiotic could be demonstrated against $M$. albicans. (See Figure 4.)

Similar results were obtained with the Rhodotorula, an organism much more sensitive to the action of Antibiotic XG. In the presence of $0.5-1.0$ unit per cc. rapid growth occurred regardless of the number of organisms present. In the presence of 2.0 units per cc., the number of organisms decreased rapidly to zero provided the initial number present was less than 10,000 per cc. It should be mentioned, however, that this does not represent a true fungicidal effect. No growth was observed in pour plates examined after 48 hours of incubation. After incubation for four or five days, however, small numbers of minute colonies appeared, indicating that the antibiotic had served only to suppress the metabolism of the organism. (See Figure 5.)

The effect of hydration on the action of Antibiotic $X G$. It has been stated previously that An-

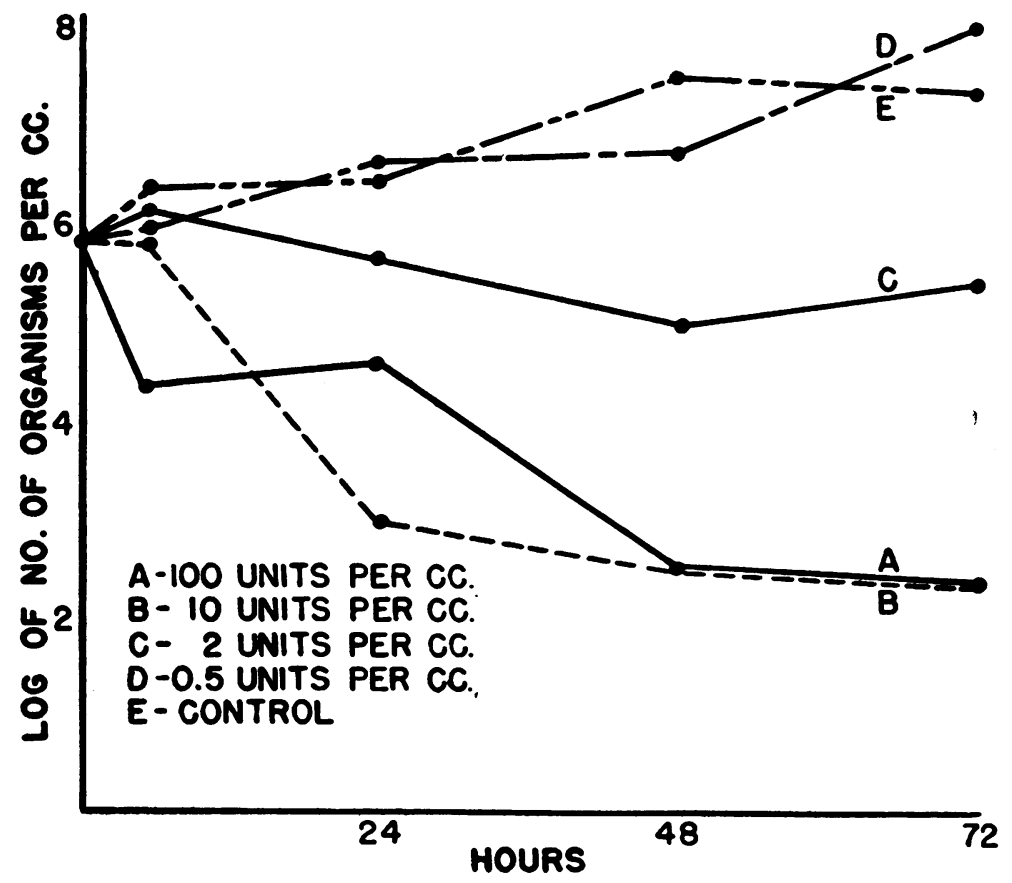

Fig. 3. The Effect of Varying Concentrations of Antibiotic XG on

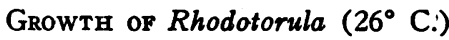




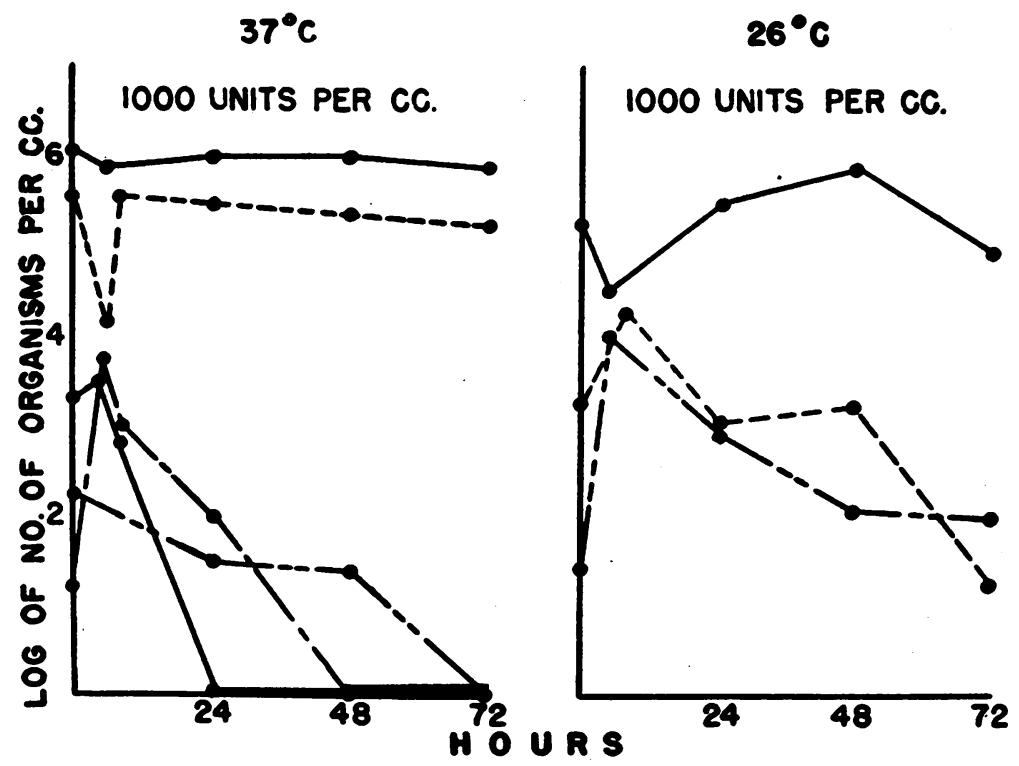

Fig. 4. The Effect of Varying Concentrations of Organisms on the Action of ANTriotic XG (Monilia albicans)

tibiotic XG on standing in water forms a hydrated gel which is biologically inactive. The difference in the activity of the dehydrated active form of the antibiotic and the hydrated gel form was readily demonstrable, as shown in Figure 6. Five hundred units of the dehydrated form per cc., although not completely inhibitory under the conditions of the experiment, were more active than
1,000 units of the hydrated or gel form per cc. The unitage of the latter form was calculated on the basis of the potency per mg. of the same preparation prior to hydration.

Toxicity

Antibiotic XG in its present degree of purity is a strongly hemolytic compound in vitro. The tox-
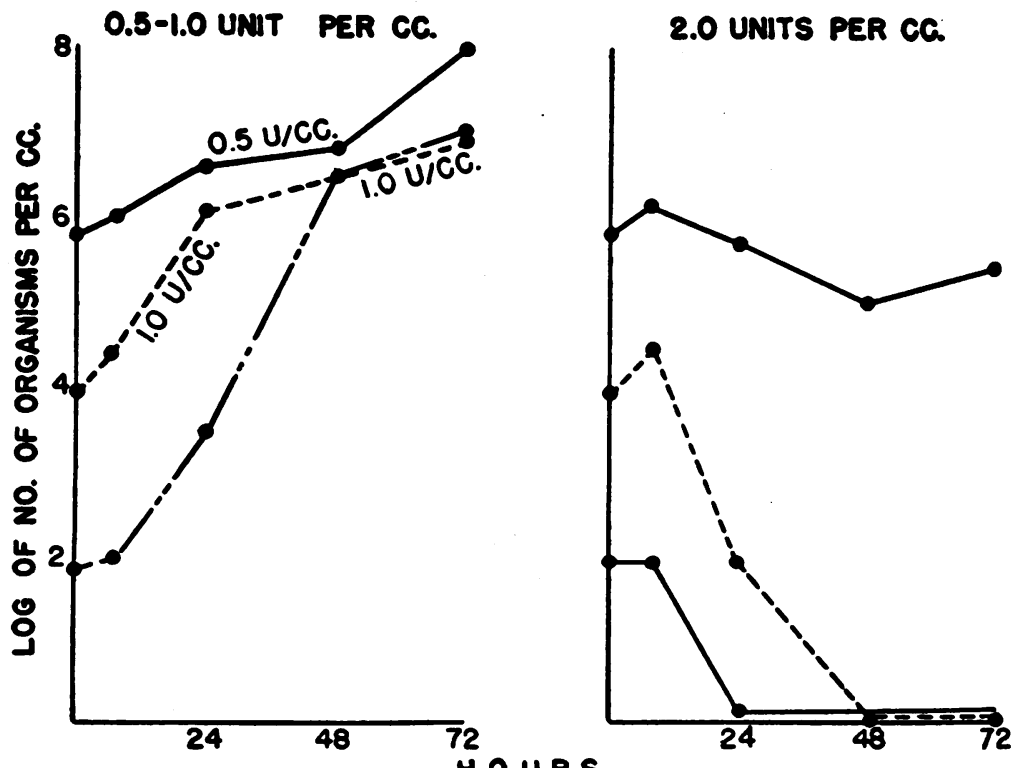

Fig. 5. The Effect of Varying Numbers of Organisms on Antifungal. Action of Antibiotic XG (Rhodotorula-26 C.) 


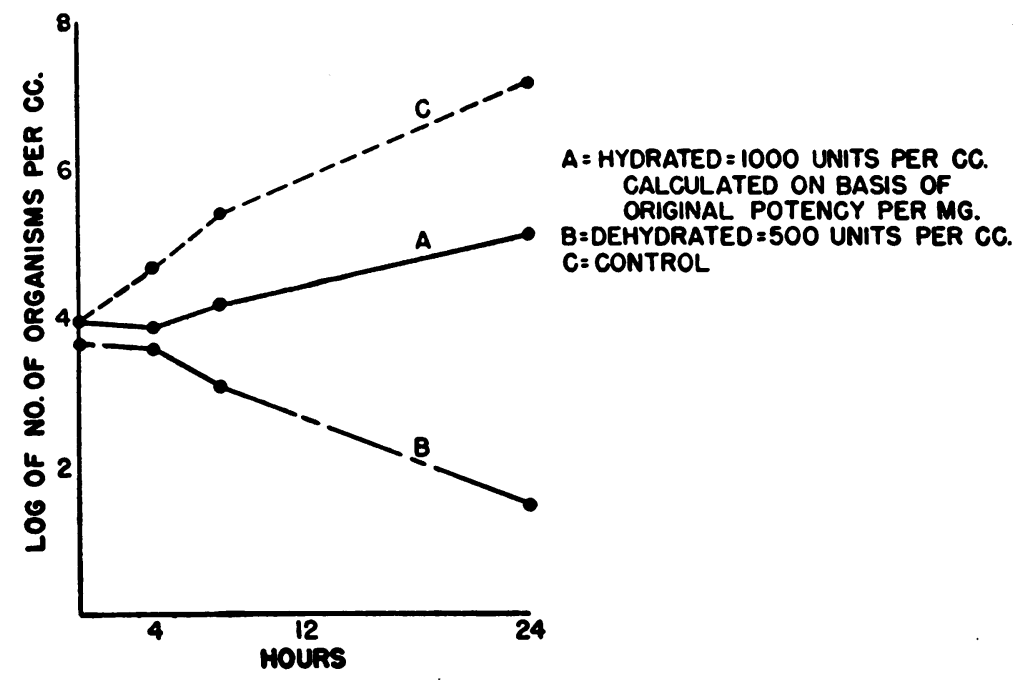

Fig. 6. The Effect of Hydration on Action of Antibiotic XG (Monilia albicans-37 $7^{\circ}$ C.)

icity of this antimicrobial agent is less than would be anticipated, nevertheless, on the basis of its hemolytic action.

The acute intravenous toxicity in mice has varied from lot to lot. The $\mathrm{LD}_{0}$ and $\mathrm{LD}_{50}$ of aqueous solutions of the most highly purified preparation tested (110 units per mg.) was 5,500 and 9,900 units respectively per $\mathrm{kg}$. mouse. The same preparation when dissolved in saline showed an acute intravenous $\mathrm{LD}_{0}$ and $\mathrm{LD}_{50}$ of 11,000 and 16,500 units respectively per $\mathrm{kg}$. mouse.

Repeated subcutaneous injections of this preparation have indicated that aqueous solutions in dosages of at least 4,100 units per kg. mouse may be administered twice daily for a period of at least five days with no toxic manifestations. (See Table III.)
Rats receiving a single subcutaneous injection of 30,000 units of a less highly purified lot per $\mathrm{kg}$. body weight showed no signs of proteinuria or other toxicity.

Rabbits receiving dosages of $5,000-10,000$ units per $\mathrm{kg}$. body weight intravenously, in aqueous solution, in most instances have died promptly after injection. The mortality may be lowered slightly by the use of saline in place of water as diluent for injection. Intravenous dosages of 5001,000 units per $\mathrm{kg}$. body weight generally cause no reactions. By the intramuscular or oral routes, rabbits tolerate readily 2,000-5,000 units per $\mathrm{kg}$. body weight in either aqueous or saline solutions.

Whether or not the antibiotic actually is absorbed following injection in these animals remains to be determined. Preliminary studies on

TABLE III

Cumulative Toxicity in Mice Antibiotic XG

\begin{tabular}{lll}
$\begin{array}{l}\text { Daily Dosage } \\
\text { Regimen }\end{array}$ & $\begin{array}{l}\text { No. of } \\
\text { Mice }\end{array}$ & $\begin{array}{l}\text { Per Cent } \\
\text { Survived }\end{array}$ \\
\hline Units/kg. mouse & & \\
\hline 4100 q.d. & 10 & 100 \\
4100 bid & 10 & 100 \\
5500 q.d. & 10 & 100 \\
5500 bid & 10 & 60
\end{tabular}

All animals were treated by the subcutaneous route for five days or until time of death. 
the absorption and excretion of Antibiotic XG have suggested that the drug must be absorbed to some extent; the concentrations of antibiotic detected in the serum, however, have been low and erratic. That the antibiotic is bound to or inactivated by plasma protein seems unlikely in view of the fact that the in vitro sensitivities of a wide variety of microorganisms to this antibiotic are not altered by the presence of serum. It seems probable that failure to detect larger quantities of Antibiotic XG in serum following parenteral administration is due to the fact that proper assay technics have not been developed to permit detection of the full amount of antibiotic present in the body fluids.

\section{Chemotherapeutic action}

It is difficult to evaluate adequately the chemotherapeutic potentialities of an antimicrobial agent such as Antibiotic XG ${ }^{*}$ by animal experimentation. In general, the fungous infections which occur in man are not readily reproduced in animals. Studies on the chemotherapeutic action of an agent such as this, therefore, can be carried out most satisfactorily directly in man.

The hemolytic action of Antibiotic XG until recently has discouraged its administration by parenteral routes. The fungous group of infections, however, represents one group of diseases in which topical therapy would take care of the great majority of cases. For this reason, Antibiotic XG is of interest despite its hemolytic action. Preliminary clinical studies on the topical and parenteral use of this agent are in progress and indicate (5)

4 Antibiotic XG possesses certain properties in common with Bacillomycin as described by Landy et al. (4). Comparative studies on the chemical nature of thise two compounds and on their biological action are in progress. that this antibiotic offers considerable promise in the topical treatment of Monilia and T. gypseum infections as well as in the treatment of a variety of other fungous diseases.

\section{CONCLUSIONS}

Antibiotic XG is a highly active antimicrobial agent which exerts a remarkable inhibitory effect on the growth of many of the pathogenic fungi. It is both fungistatic and fungicidal, depending on the experimental conditions used. Its activity is influenced by the number of organisms present, the rate of growth of the organisms, the concentration of antibiotic, and the temperature of incubation. In its present degree of purity, it is hemolytic in nature. Preliminary studies indicate a relatively low degree of toxicity, however, and suggest that with purification and further understanding of the mechanism of its action, it may be possible to administer it parenterally. In its present form, Antibiotic XG offers great promise as a chemotherapeutic agent for the treatment of a group of infections in which local therapy usually is adequate.

\section{BIBLIOGRAPHY}

1. Lewis, G. M., Hopper, M. E., and Shultz, S., In vitro fungistasis by bacterium (Bacillus subtilis var. XG, XY). Arch. Dermat. \& Syph., 1946, 54, 300.

2. Lenert, T. F., and Hobby, G. L., Observations on the action of streptomycin (I). Proc. Soc. Exper. Biol. \& Med., 1947, 65, 235.

3. Hobby, G. L., and Lenert, T. F., Observations on the action of streptomycin in vitro (II). Proc. Soc. Exper. Biol. \& Med., 1947, 65, 242.

4. Landy, M., Warren, G. H., Rosenman, S. B., and Colio, L. G., Bacillomycin: An antibiotic from Bacillus subtilis active against pathogenic fungi. Proc. Soc. Exper. Biol. \& Med., 1948, 67, 539.

5. Lewis, G. H., Personal communication, 1949. 